INPLASY

PROTOCOL

To cite: Zheng et al. A metaanalysis of the influence of Chinese patent medicine Guizhi Fuling Pills on sex hormone levels and pregnancy outcome in patients with polycystic ovary syndrome. Inplasy protocol 202160045. doi:

10.37766/inplasy2021.6.0045

Received: 14 June 2021

Published: 14 June 2021

Corresponding author:

Huizhi Zheng

huizhi301@126.com

Author Affiliation:

Nanchong Central Hospital

Support: No.YAZX19-Y-12.

Review Stage at time of this submission: Piloting of the study selection process.

Conflicts of interest:

None declared.

\section{A meta-analysis of the influence of Chinese patent medicine Guizhi Fuling Pills on sex hormone levels and pregnancy outcome in patients with polycystic ovary syndrome}

\author{
Zheng, HZ1; Yang, M2; Zhao, R³; Qiang, HM4; Tang, JW5.
}

Review question / Objective: 1. Does Guizhi Fuling Pills improve sex hormone levels in patients with polycystic ovarian syndrome? 2. Does Guizhi Fuling Pills improve pregnancy outcome in patients with polycystic ovarian syndrome? 3. Whether duration has impact on intervention effects? 4. How about the safety of Guizhi Fuling Pills in the treatment of polycystic ovarian syndrome?

Condition being studied: Polycystic ovary syndrome is a common endocrine disorder in women of adolescence and childbearing age, characterized by oligomenorrhea, hirsutism, acne, and obesity. Guizhi Fuling Pills are from Zhang Zhongjing's "Golden Chamber Synopsis". The original prescription used to treat women's symptoms that caused more than pregnancy leakage or fetal movement. It was widely used in various diseases in later generations. In recent years, some scholars have applied Guizhi Fuling Pills to PCOS patients and found that it has the effect of increasing ovulation rate and pregnancy rate. This study will explore the effects of Guizhi Fuling Pills on ovulation rate, pregnancy rate and sex hormone levels in patients with polycystic ovary syndrome.

INPLASY registration number: This protocol was registered with the International Platform of Registered Systematic Review and Meta-Analysis Protocols (INPLASY) on 14 June 2021 and was last updated on 14 June 2021 (registration number INPLASY202160045).

\section{INTRODUCTION}

Review question / Objective: 1. Does Guizhi Fuling Pills improve sex hormone levels in patients with polycystic ovarian syndrome? 2. Does Guizhi Fuling Pills improve pregnancy outcome in patients with polycystic ovarian syndrome? 3 . Whether duration has impact on intervention effects? 4. How about the safety of Guizhi Fuling Pills in the treatment of polycystic ovarian syndrome? 
Condition being studied: Polycystic ovary syndrome is a common endocrine disorder in women of adolescence and childbearing age, characterized by oligomenorrhea, hirsutism, acne, and obesity. Guizhi Fuling Pills are from Zhang Zhongjing's "Golden Chamber Synopsis". The original prescription used to treat women's symptoms that caused more than pregnancy leakage or fetal movement. It was widely used in various diseases in later generations. In recent years, some scholars have applied Guizhi Fuling Pills to PCOS patients and found that it has the effect of increasing ovulation rate and pregnancy rate. This study will explore the effects of Guizhi Fuling Pills on ovulation rate, pregnancy rate and sex hormone levels in patients with polycystic ovary syndrome.

\section{METHODS}

Search strategy: We will search the following electronic databases: PubMed, Embase, Cochrane Library, Web of Science, CNKI, WanFang data, VIP and CBM. The date will be restricted from 2000 to 2021.

\section{Participant or population: Polycystic ovary} syndrome.

Intervention: Guizhi Fuling Pills.

Comparator: Western medicine with clear curative effect, blank control, placebo.

Study designs to be included: Randomized controlled trial.

Eligibility criteria: (1) Participants: patients with polycystic ovary syndrome; (2) Intervention: Chinese patent medicine; (3) Control: western medicine with clear curative effect, blank control, placebo; (4) Outcomes: pregnancy rate, ovulation rate, incidence of adverse reactions, $\mathrm{FSH}, \mathrm{LH}$, testosterone, estradiol, prolactin, LH/FSH ; (5) Study design: randomized controlled trials; (6) Language: English and Chinese.

Information sources: The PubMed, EMBASE, Web of Science, Cochrane Library, CNKI, Wanfang, VIP and CBM will be systematically searched for this review with language restriction to English and Chinese. Other restrictions will be imposed on publication time from 2000 to 2021. Search methods of MeSH terms with free words will be adopted in English databases. In addition, some unpublished studies and other relevant literature will be identified through manual searching.

Main outcome(s): pregnancy rate; ovulation rate; incidence of adverse reactions.

Additional outcome(s): FSH; LH; Testosterone; Estradiol; Prolactin; LH/FSH.

Quality assessment / Risk of bias analysis: The risk of bias of eligible studies will be assessed by using the Cochrane riskofbias assessment tool. According to this tool, the risk of bias of study is assessed from 7 items: random sequence generation and allocation concealment (selection bias), blinding of participants and personnel (performance bias), blinding of outcome assessment (detection bias), incomplete outcome data (attrition bias), selective reporting (reporting bias) and other bias.

Strategy of data synthesis: We will conduct quantitative analysis if the outcome indicators include more than two studies, otherwise use narrative analysis. As for continuous variables, standardized mean difference (SMD) will be used to express the effect sizes. As for dichotomous variables, risk ratio (RR) will be used to express the effect sizes. All included studies will be combined using a random effects meta-analysis.

Subgroup analysis: If there is substantial heterogeneity between studies, then subgroup analysis will be conducted to investigate the sources of heterogeneity. The grouping factor for subgroup analysis is as follows: duration of Guizhi Fuling Pills.

Sensitivity analysis: We will omit each study that is included in meta-analysis one by one if there are sufficient studies.

Country(ies) involved: China. 
Keywords: Guizhi Fuling Pills; Polycystic ovary syndrome; Sex hormone; Pregnancy outcome; Meta-analysis.

Contributions of each author:

Author 1 - Huizhi Zheng - Document retrieval, formal analysis, software and writing-original draft will be conducted by Author one.

Email: huizhi301@126.com

Author 2 - Mei Yang - Data curation and formal analysis will be conducted by Author two.

Email: 939952424@qq.com

Author 3 - Rong Zhao - Data curation and formal analysis will be conducted by Author three.

Email: 7766903@qq.com

Author 4 - Hongmei Qiang - Writing-original draft will be conducted by Author four.

Email: 50597947@qq.com

Author 5 - Junwei Tang - Writing-Review and editing will be conducted by Author five.

Email: 1017633308@qq.com 